Draft Version OCtober 10, 2018

Preprint typeset using $\mathrm{L}^{A} \mathrm{~T}_{\mathrm{E}} \mathrm{X}$ style emulateapj v. 08/22/09

\title{
SEARCH FOR CHAOS IN NEUTRON STAR SYSTEMS: IS CYG X-3 A BLACK HOLE?
}

\author{
Bidya Binay KaraK ${ }^{1}$, Jayanta Dutta ${ }^{2}$, Banibrata Mukhopadhyay ${ }^{3}$ \\ Astronomy and Astrophysics Program, Department of Physics, Indian Institute of Science, Bangalore 560012, India \\ Draft version October 10, 2018
}

\begin{abstract}
The accretion disk around a compact object is a nonlinear general relativistic system involving magnetohydrodynamics. Naturally the question arises whether such a system is chaotic (deterministic) or stochastic (random) which might be related to the associated transport properties whose origin is still not confirmed. Earlier, the black hole system GRS 1915+105 was shown to be low dimensional chaos in certain temporal classes. However, so far such nonlinear phenomena have not been studied fairly well for neutron stars which are unique for their magnetosphere and $\mathrm{kHz}$ quasi-periodic oscillation (QPO). On the other hand, it was argued that the QPO is a result of nonlinear magnetohydrodynamic effects in accretion disks. If a neutron star exhibits chaotic signature, then what is the chaotic/correlation dimension? We analyze RXTE/PCA data of neutron stars Sco X-1 and Cyg X-2, along with the black hole Cyg X-1 and the unknown source Cyg X-3, and show that while Sco X-1 and Cyg X-2 are low dimensional chaotic systems, Cyg X-1 and Cyg X-3 are stochastic sources. Based on our analysis, we argue that Cyg X-3 may be a black hole.

Subject headings: stars: neutron - X-rays: binaries - X-rays: individual (Sco X-1, Cyg X-1,2,3) -
\end{abstract} accretion, accretion disks

\section{INTRODUCTION}

X-ray binary systems vary on timescales ranging from months to milli-seconds (see, e.g., (Chen et al. 1997; Paul et al. 1997; Nowak et al. 1999; Cui 1999; Gleissner et al. 2004; Axelsson 2008)). Detailed analysis of their temporal variability and fluctuation provides important insights into the geometry and physics of emitting regions and the accretion process. However, the origin of variability is still not clear. It could be due to varying external parameters, like the infalling mass accretion rate. It could also be due to possible instabilities in the inner regions of the accretion disk where the flow is expected to be nonlinear and turbulent. Uttley et al. (2005) (see also Timmer et al. 2000 and Thiel et al. 2001) argued that the non-linear behavior of a system can be understood from the log-normal distribution of the fluxes and the rms-flux relation. This implies that the temporal behavior of the system may be driven by underlying stochastic variations. By studying the underlying nonlinear behavior, important constraints can be obtained on these various possibilities.

An elegant way of obtaining the constraint is to perform the nonlinear time series analysis of observed data and to compute the correlation dimension $D_{2}$ in a nonsubjective manner. This technique has already been used to diverse situations ( Grassberger \& Procaccia 1983a; Grassberger \& Procaccia 1983b; $\quad$ Schreiber 1999; Aberbandel 1996; Serre et al. 1996; Misra et al. 2004; Misra et al. 2006; Harikrishnan et al. 2006), and references therein). By obtaining $D_{2}$ as a function of the embedding dimension $M$, one can infer the origin of the variability. For example, $D_{2} \approx M$ for all $M$ corresponds to the system having stochastic fluctuation which favors

\footnotetext{
1 bidya_karak@physics.iisc.ernet.in

2 dutta@physics.iisc.ernet.in

3 bm@physics.iisc.ernet.in
}

the idea that X-ray variations are driven by variations of some external parameters. On the other hand, a saturated $D_{2}$ to a finite (low) value, beyond a certain $\mathrm{M}$, implies a deterministic chaos which argues in favor of inner disk instability. However, to implement the algorithm successfully, the system in question should provide enough data.

The technique was used earlier to understand the nonlinear nature of a black hole system Cyg X1 (Unno et al. 1990) and an Active Galactic Nucleus (AGN) Ark 564 (Gliozzi et al. 2002), but due to insufficient data points the analyses were hampered and no concrete conclusions were made about $D_{2}$. Later on, another black hole system GRS 1915+105 was analyzed (Misra et al. 2004; Misra et al. 2006; Harikrishnan et al. 2006) which was shown to display low dimensional chaos in certain temporal classes, while stochastic in other classes.

However, so far none of the neutron star systems have been analyzed in detail in order to understand the origin of nonlinearity. Decades back, Voges et al. (1987) attempted to understand the chaotic nature of Her X-1, but the analysis was hampered by low signal to noise ratio (Norris \& Matilsky 1989). Since then the investigation of chaotic signature in neutron stars remains unattended. Can a neutron star system not be deterministic? Indeed several features of X-ray binary systems consisting of a neutron star, such as their magnetosphere and kHz Quasi-Periodic Oscillation (QPO) and its possible relation to the spin frequency of the neutron star, favor the idea that they exhibit nonlinear resonance (e.g. (Blaes et al. 2007; Mukhopadhyay 2009)). While the QPO itself is a mysterious feature whose origin is still unclear, its possible link to the spin frequency of the neutron star $^{4}$ indicates the origin

\footnotetext{
${ }^{4}$ However, some authors (Mendez \& Belloni 2007) suggested that the $\mathrm{kHz}$ QPOs may not be related to the spin.
} 
of QPO to be from nonlinear phenomena. Several LMXBs having a neutron star exhibit twin $\mathrm{kHz}$ QPOs (Mendez et al. 1998; van der Klis 2006). For some of them, e.g. 4U 1636-53 (Jonker et al. 2002), KS 1731-260 (Smith et al. 1997), 4U 1702429 (Markwardt et al. 1999), $4 \mathrm{U} \quad$ 1728-34 (van Straaten et al. 2002), the spin frequency of the neutron star has been predicted from observed data. However, for the source Sco X-1, which exhibits noticeable time variability (Mendez \& van der Klis 2000), while we observe twin $\mathrm{kHz}$ QPOs, we do not know the spin frequency yet (but see (Mukhopadhyay 2009)). For another neutron star Cyg X-2, we do observe $\mathrm{kHz}$ QPOs (Wijnands et al. 1998) as well. Several black holes also exhibit QPOs, e.g. GRS $1915+105$ (Belloni et al. 2001; McClintock \& Remillard 2006), Cyg X-1 (Angelini et al. 1994).

In the present paper, we first aim at analyzing the time series of two neutron star sources Sco X-1 and Cyg X-2 to understand if a neutron star is a deterministic nonlinear (chaotic) system. Then we try to manifest the knowledge of nonlinear (chaotic/random) property of compact sources to distinguish a black hole from a neutron star. Subsequently, knowing their difference based on the said property, we try to identify the nature of a unknown source (whether it is a black hole or a neutron star). While the nature of some sources, as mentioned above, has already been predicted based on alternate method, for some others, e.g. Cyg X-3, SS433, it has not yet been confirmed.

For the present purpose, we therefore concentrate on three additional sources Cyg X-1, Cyg X-2 and Cyg X-3. While Cyg X-1 has been predicted to be a black hole and Cyg X-2 be a neutron star, the nature of Cyg X-3 is not confirmed yet. Some authors (Ergma \& Yungelson 1998; Schmutz et al. 1996; Szostek \& Zdziarski 2008) argued for a black hole nature of $\mathrm{Cyg} \mathrm{X}-3$, on the basis of its jet, the time variations in the infrared emission lines, the BeppoSAX X-ray spectra and so on. However, earlier it was argued for a neutron star (Chadwick et al. 1985) by measuring its $1000 \mathrm{GeV} \gamma$-rays which suggests a pulsar period of $12.5908 \pm 0.0003 \mathrm{~ms}$. By analyzing the time series and computing the correlation dimension $D_{2}$, here we aim at pinpointing the nature of Cyg X-3: whether a black hole or a neutron star.

In the next section, we briefly outline the procedure to be followed in understanding the nonlinear nature of a compact object from observed data and to implement it to analyze the neutron star source Sco X-1. In $\S 3$, we then describe nonlinear behaviors of Cyg X-1, Cyg X-2 and Cyg X-3. Subsequently, in $\S 4$, we compare all the results and argue for a black hole nature of Cyg X-3. Finally, we summarize in $\S 5$.

\section{PROCEDURE AND NONLINEAR NATURE OF SCO X-1}

The method to obtain $D_{2}$ is already established (see, e.g., (Grassberger \& Procaccia 1983b; Misra et al. 2004; Harikrishnan et al. 2006)). Therefore, here we discuss it briefly. We consider PCA data of the RXTE satellite (see Table 1 for the observations IDs) from the archive for our analysis. We process the data using the FTOOLS software. We extract a few continuous data streams of $2500-3500 \mathrm{sec}$ long. The time resolution used to generate lightcurves is $\sim 0.1-1 \mathrm{sec}$. This is the range of optimum resolution, at least for the sources we consider, to minimize noise without losing physical information of the sources. A finer time resolution would be Poisson noise dominated and a larger binning might give too few data points to derive physical parameters from it (see Misra et al. 2004, 2006, for details).

Then we calculate the correlation dimension according to the Grassberger \& Procaccia (1983a,b) algorithm. From the time series $s\left(t_{i}\right)(\mathrm{i}=1,2, \ldots, \mathrm{N})$, we construct an $\mathrm{M}$ dimensional space (called embedding space), in which any vector has the following form:

$$
x\left(t_{i}\right)=\left[s\left(t_{i}\right), s\left(t_{i}+\tau\right), \ldots \ldots \ldots, s\left(t_{i}+(M-1) \tau\right)\right],
$$

where $\tau$ is the time delay chosen in such a way that each component of the vector $x\left(t_{i}\right)$ is independent of each other. For a particular choice of embedding dimension $M$, we compute the correlation function:

$$
C_{M}(r)=\frac{1}{N\left(N_{c}-1\right)} \sum_{i=1}^{N} \sum_{j=1, j \neq i}^{N_{c}} \Theta\left(r-\left|x_{i}-x_{j}\right|\right),
$$

which is basically the average number of points within a hypersphere of diameter $r$, where $\Theta$ is a Heaviside step function, $N$ the total number of points and $N_{c}$ the number of centers. If the system has a strange attractor, then one can show that for a small value of $r$

$$
D_{2}(M)=\frac{d \log C_{M}(r)}{d \log r} .
$$

In this numerical calculation, we divide the whole phase space into $M$ cubes of length $r$ around a point and we count the average number of data points in these cubes to calculate $C_{M}(r)$. The edge effects, which come due to the finite number of data points, have been avoided by calculating $C_{M}(r)$ in the range $r_{\text {min }}<r<$ $r_{\max }$, where $r_{\min }$ is the value of $r$ for $C_{M}(r)$ just greater than one and $r_{\max }$ can be found by restricting the $M$ cubes to be within the embedding space. In Fig. 11 we show the variation of $\log \left(C_{M}(r)\right)$ with $\log (r)$ for different values of $M$ for Sco X-1 data.

$D_{2}(M)$ can be calculated from the linear part of the $\log \left(C_{M}(r)\right)$ vs. $\log (r)$ curve and its value depends on the value of $M$. For a stochastic system, $D_{2} \approx M$ for all $M$. On the other hand, for a chaotic or deterministic system, initially $D_{2}(M)$ increases linearly with the increase of $M$, then it reaches a certain value and saturates. This saturated value of $D_{2}$ is taken to be the correlation dimension of the system which is a non-integer. The standard deviation gives the error in $D_{2}$.

We first concentrate upon the neutron star source Sco X-1. In Figs. 2a,b we show respectively the lightcurve and the variation of $D_{2}$ as a function of $M$ and find that $D_{2}$ saturates to a value $2.6( \pm 0.8)$. As this is a non-integer, the system might be chaotic. On the other hand, we know that the Lorenz attractor is an example of an ideal chaos with $D_{2}=2.05$. Therefore, Sco X-1 may be like a Lorenz system. But due to noise its $D_{2}$ seems appearing slightly higher (Misra et al. 2004; Misra et al. 2006) than the actual value. However, one should be cautious about the fact that Sco X-1 is a bright source (much brighter than other sources considered later). Hence, the dead time effect on 
the detector might affect the actual value of saturated $D_{2}$ and the computed value might be slightly different than the actual one. However, this can not rule out the signature of chaos in Sco X-1, particularly because the corresponding count rates are confined in the same order of magnitude and hence the dead time effect, if any, is expected to affect all the count rates in a similar way.

However, a saturated $D_{2}$ is necessary but not a sufficient evidence for chaos. Existence of color noise (for which the power spectrum $P(\nu) \propto \nu^{-\alpha}$, where the power spectral indices $\alpha=0,1$ and 2 correspond to "white", "pink" and "red" noise respectively) into a stochastic system might lead to a saturated $D_{2}$ of low value as well (e.g. Osborne \& Provenzale 1989; Theiler et al 1992; Misra et al. 2006; Harikrishnan et al. 2006). Therefore, it is customary to analyze data by alternate $\operatorname{approach}(\mathrm{s})$ to distinguish it from a pure noisy time series (Kugiumtzis 1999). One of the techniques is the surrogate data analysis (e.g. (Schreiber \& Schmitz 1996)), which has been described earlier in detail and implemented for a black hole (Misra et al. 2006; Harikrishnan et al. 2006). In brief, surrogate data is random data generated by taking the original signal and reprocessing it so that data has the same/similar Fourier power spectrum and autocorrelation along with the same distribution, mean and variance as of the original data, but has lost all deterministic characters. Then the same analysis is carried out to the original data and the surrogate data to identify any distinguishable feature(s) between them. The scheme proposed by Schreiber \& Schmitz (1996), known as Iterative Amplitude-Adjusted Fourier Transform (IAAFT), is more consistent to generate surrogate data.

Figures 2 c-f compare results for the original data with the surrogate data. It is clear that while distributions and power spectra are same/similar for both the data sets, $D_{2}$ is much higher for the surrogate data which suggests existence of low dimensional chaos in Sco X-1 with $D_{2} \sim 2.6$. This confirms, for the first time to best of our knowledge, a neutron star source to display chaotic behavior. As the existence of chaos is a plausible signature of instability in the inner region of accretion flows which is nonlinear and turbulent, as mentioned in $\S 1$, the corresponding QPO, which is presumably an inner disk phenomenon as well, is expected to be governed by nonlinear resonance mechanisms (e.g. Mukhopadhyay 2009).

In Table 1, we enlist the average counts $\langle S\rangle$, its root mean square $(r m s)$ variation $\sqrt{\left.\left\langle S^{2}\right\rangle-<S\right\rangle^{2}} /<$ $S\rangle$, the expected Poisson noise $\langle P N\rangle \equiv \sqrt{\langle S\rangle}$, and the ratio of the expected Poisson noise to the $\mathrm{rms}$ value for all sources. It clearly shows a strong correlation between the inferred behavior of the systems and the ratio of the expected Poisson noise to the $\mathrm{rms}$ value.

\section{NONLINEARITY OF CYG X-1,2,3}

We now look into three additional compact sources: Cyg X-1 (black hole), Cyg X-2 (neutron star) and Cyg X3 (nature is not confirmed yet), and apply the same analysis as in the case of Sco X-1. Figure $3 \mathrm{~b}$ shows that $D_{2}$ for Cyg X-1 seems not to saturate and appears very high. However, there is no surprise in it because its variability is similar to the temporal class $\chi$ of the black hole GRS $1915+105$ which was shown to be Poisson noise dominated and stochastic in nature
(Misra et al. 2004). Indeed, earlier analysis of Cyg X-1 data, while it could not conclusively quantify the underlying chaotic behavior due to insufficient data, revealed very high dimensional chaos. Moreover a large $\langle P N\rangle$ (as well $\langle P N>/$ rms) for Cyg X-1, compared to that for Sco X-1 given in Table 1, reveals the system to be noise dominated. This ensures $\mathrm{Cyg} \mathrm{X}-1$ to be different from Sco X-1. However, the variation of $D_{2}$ as a function of $M$ for the original data does not deviate noticeably from that of corresponding surrogate data, as shown in Fig. 3r, which argues that $\mathrm{Cyg} \mathrm{X}-1$ is not a chaotic system.

Figures $40, \mathrm{c}$ show that $D_{2}$ for Cyg X-2 saturates to a low value $\sim 4$, which is significantly different than that of corresponding surrogate data. The power spectra and distributions, on the other hand, for original and surrogate data are same/similar (as shown in Sco X-1, not repeated further). The saturated $D_{2}$ for Cyg X-2 is almost double than that of Lorenz system, possibly due to high Poisson noise to rms ratio (see Table-1). This suggests the corresponding system to be a low dimensional chaos.

From Figs. 5b,c we see that for Cyg X-3 the variations of $D_{2}$ as a function of $M$ for original and surrogates data are similar to that of Cyg X-1. This confirms that the behavior of the unknown source $\mathrm{Cyg} \mathrm{X}-3$ is similar to that of the black hole source Cyg X-1 (see, however, (Axelsson et al. 2008)). Note from Table 1 that $\mathrm{Cyg} \mathrm{X}-1$, $\mathrm{X}-2, \mathrm{X}-3$ are significantly noise dominated compared to Sco X-1. Although noise could not suppress the chaotic signature in the neutron star Cyg X-2, its saturated $D_{2}$ is higher than that of Sco X-1. On the other hand, even though the Poisson noise to rms ratio in Cyg X-1 is lower than that in Cyg X-2 (but Poisson noise itself is higher in Cyg X-1), its $D_{2}$ never saturates, which confirms the source to be non-chaotic; the apparent stochastic signature is not due to Poisson noise present into the system.

\section{COMPARISON BETWEEN CYG X-1, CYG X-2 AND CYG X-3}

Finally, we compare the variations of $D_{2}$ for all three cases of Cygnus in Fig. 6. Remarkably we find that $D_{2}$ values for Cyg X-1 and Cyg X-3 practically overlap, appearing much larger compared to that for $\mathrm{Cyg} \mathrm{X}-2$ which is shown to be a low dimensional chaotic source. On the other hand, Cyg X-2 is a confirmed neutron star and Cyg X-1 a black hole. Therefore, Cyg X-3 may be a black hole.

\section{SUMMARY}

The source Cyg X-3, whose nature is not confirmed yet, seems to be a black hole based on the analysis of its nonlinear behavior. On the other hand, we have shown, for the first time to best of our knowledge, that neutron star systems could be chaotic in nature. The signature of deterministic chaos, which argues in favor of inner disk instability, into an accreting system has implications in understanding its transport properties particularly in Keplerian accretion disk (Winters et al. 2003). Note that in Keplerian accretion disks transport is necessarily due to turbulence in absence of significant molecular viscosity. The signature of chaos confirms instability and then plausible turbulence. On the other hand, for a rotating neutron star having a magnetosphere, signature of chaos 
suggests their QPOs to be nonlinear resonance phenomena (Mukhopadhyay 2009). The absence of chaos and related/plausible signature of instability in Cyg X-1 and Cyg X-3 suggests the underlying accretion disk to be sub-Keplerian (Narayan \& Yi 1995; Chakrabarti 1996) in nature which is dominated significantly by gravitational force.
This work is partly supported by a project (Grant No. SR/S2/HEP12/2007) funded by Department of Science and Technology (DST), India. Also the financial support to one of the authors (JD) has been acknowledged. The authors would like to thank Arnab Rai Choudhuri of IISc and the anonymous referee for carefully reading the manuscript, constructive comments and suggestions.

\section{REFERENCES}

Aberbandel, H. D. L. 1996, Analysis of Observed Chaotic data (Springer: New York)

Angelini, L., White, N. E., Stella, L. 1994, in New Horizon of X-Ray Astronomy, ed. F. Makino, \& T. Ohashi (Tokyo: Universial Academy Press), 429

Axelsson, M. 2008, AIPC, 1054, 135

Axelsson, M., Larsson, S. \& Hjalmarsdotter, L. 2008, MNRAS, 394, 1544

Belloni, T., Méndez, M., Sánchez-Fernández, C. 2001, ApJ, 372, 551

Blaes, O. M., Srmková, E., Abramowicz, M. A., Kluniak, W., Torkelsson, U. 2007, ApJ, 665, 642

Chadwick, P. M., Dipper, N. A., Dowthwaite, J. C., Gibson, A. I. \& Harrison, A. B. 1985, Nature, 318, 642

Chakrabarti, S. K. 1996, ApJ, 464, 664

Chen, X., Swank, J. H. \& Taam, R. E. 1997, ApJ, 477, L41.

Cui, W. 1999, ApJ, 524, L59

Ergma, E. \& Yungelson, L. R. 1998, A\&A, 333, 151

Gleissner, T., Wilms, J., Pottschmidt, K., Uttley, P., Nowak, M. A., \& Staubert, R. 2004, A\&A, 414, 1091

Gliozzi, M., Brinkmann, W., Räth, C., Papadakis, I. E., Negoro, H. \& Scheingraber, H. 2002, A\&A, 391, 875

Grassberger, P. \& Procaccia, I. 1983, Physica D, 9, 189

Grassberger, P. \& Procaccia, I 1983, Phys. Rev. Lett., 50, 346

Harikrishnan, K. P., Misra, R., Ambika, G. \& Kembhavi, A. K. 2006, Physica D, 215, 137

Jonker, P. G., Mendez, M., \& van der Klis, M. 2002, MNRAS, 336, L1

Kugiumtzis, D. 1999, Phys. Rev. E, 60, 2808

Markwardt, Craig B., Strohmayer, Tod E. \& Swank, Jean H, 1999, ApJ 512, L125

McClintock, J. E., \& Remillard, R. A. 2006, in Compact Stellar X-Ray Sources, ed. W. H. G. Lewin \& M. van der Klis, (Cambridge: Cambridge Univ. Press)

Mendez, M., \& Belloni, T. 2007, MNRAS, 381, 790

Mendez, M., van der Klis, M., Wijnands, R., Ford, E. C., van Paradijis, J., \& Vaughan, B. A. 1998, ApJ, 505, L23

Mendez, M. \& van der Klis 2000, MNRAS 318, 938

Misra, R., Harikrishnan, K. P., Ambika, G. \& Kembhavi, A. K. 2006, ApJ, 643, 1114
Misra, R., Harikrishnan, K. P., Mukhopadhyay, B., Ambika, G. \& Kembhavi, A. K. 2004, ApJ, 609, 313

Mukhopadhyay, B. 2009, ApJ, 694, 387

Narayan, R. \& Yi, I. 1995, ApJ, 452, 710

Norris, J. P. \& Matilsky, T. A. 1989, ApJ, 346, 912

Nowak, M. A., Vaughan, B. A., Wilms, J., Dove, J. B. \& Begelman, M. C. 1999, ApJ, 510, 874

Osborne, A. R. \& Provenzale, A. 1989, Phy. D, 35, 357

Paul, B., Agrawal, P. C., Rao, A. R., Vahia, M. N., Yadav, J. S., Marar, T. M. K., Seetha, S., Kasturirangan, K. 1997, A\&A 320 L37

Schmutz, W., Geballe, T. R. \& Schild, H. 1996, A\&A, 311, 25

Schreiber, T. 1999, Phys. Rep., 308, 1

Schreiber, T. \& Schmitz, A. 1996, Phys. Rev. Lett., 77, 635

Serre, T., Kollath, Z. \& Buchler, J. R. 1996, A\&A, 311, 833

Smith, D. A., Morgan, E. H., \& Bradt, H. 1997, ApJ, 479, 137

Szostek, A. \& Zdziarski, A. A. 2008, MNRAS, 386, 593

Theiler, J., Eubank, S., Longtin, A., Galdrikian, B., Doyne, F. J. 1992, Physica D, 58, 77

Unno, W., et al. 1990, PASJ, 42, 269

Timmer, J, Schwarz, U \& Voss, H. U et al. 2000, Phys. Rev. E, 61,1342

Thiel, M., Romano, M \& Schwarz, U et al. 2001, A\&A suppl. 276, 187

Uttley, P., McHardy, I. M., Vaughan, S. 2005, MNRAS, 359, 345 van der Klis, M. 2006, AdSpR, 38, 2675

van Straaten, S., van der Klis, M., di Salvo, T., \& Belloni, T. 2002, ApJ, 568, 912

Voges, W., Atmanspacher, H., \& Scheingraber, H. 1987, ApJ, 320,794

Winters, W. F., Balbus, S. A., \& Hawley, J. F. 2003, MNRAS, 340,519

Wijnands, R., Homan, J., van der Klis, M., Kuulkers, E., van Paradijs, J., Lewin, W. H. G., Lamb, F. K., Psaltis, D. \& Vaughan, B. 1998, ApJ, 493, L87 


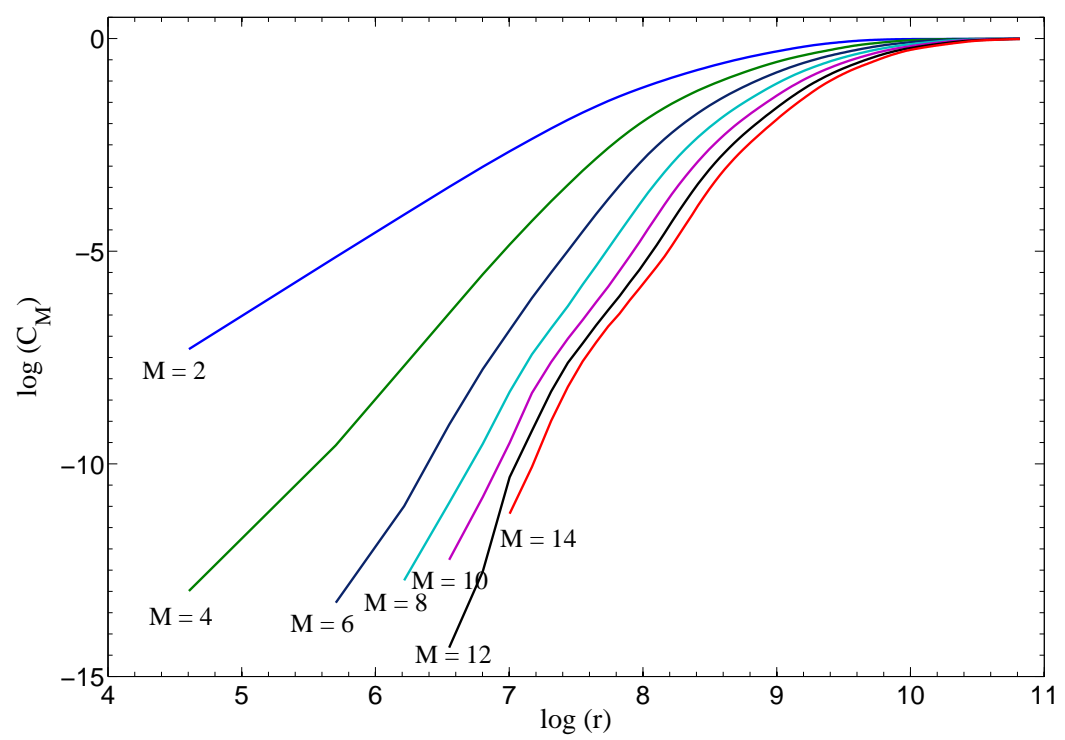

FIG. 1.- Variation of $\log \left(C_{M}\right)$ as a function of $\log (r)$ for different embedding dimensions. The linear scaling range is used to calculate the correlation dimension.

TABLE 1

OBSERVED DATA

\begin{tabular}{ccccccc}
\hline \hline Source & Obs. I. D. & $\langle S\rangle$ & $r m s$ & $\langle P N\rangle$ & $\langle P N\rangle / r m s$ & Behavior \\
\hline \hline Sco X-1 & $91012-01-02-00$ & 58226 & 0.074 & 0.004 & 0.054 & $\mathrm{C}$ \\
\hline Cyg X-1 & $10512-01-09-01$ & 10176 & 0.261 & 0.031 & 0.119 & $\mathrm{NC} / \mathrm{S}$ \\
\hline Cyg X-2 & $10063-10-01-00$ & 4779 & 0.075 & 0.014 & 0.191 & $\mathrm{C}$ \\
\hline Cyg X-3 & $40061-01-07-00$ & 3075 & 0.125 & 0.057 & 0.455 & $\mathrm{NC} / \mathrm{S}$ \\
\hline \hline
\end{tabular}

Columns:- 1: Name of the source, 2: RXTE Observational identification number from which the data has been extracted. 3: The average count in the lightcurve $\langle S\rangle$ : The root mean square variation in the lightcurve, rms. 5: The expected Poisson noise variation, $\langle P N\rangle \equiv \sqrt{\langle S\rangle}$. 6: The ratio of the expected Poisson noise to the actual root mean square variation 7: The behavior of the system (C: chaotic behavior; S: stochastic behavior; NC: nonchaotic behavior) 

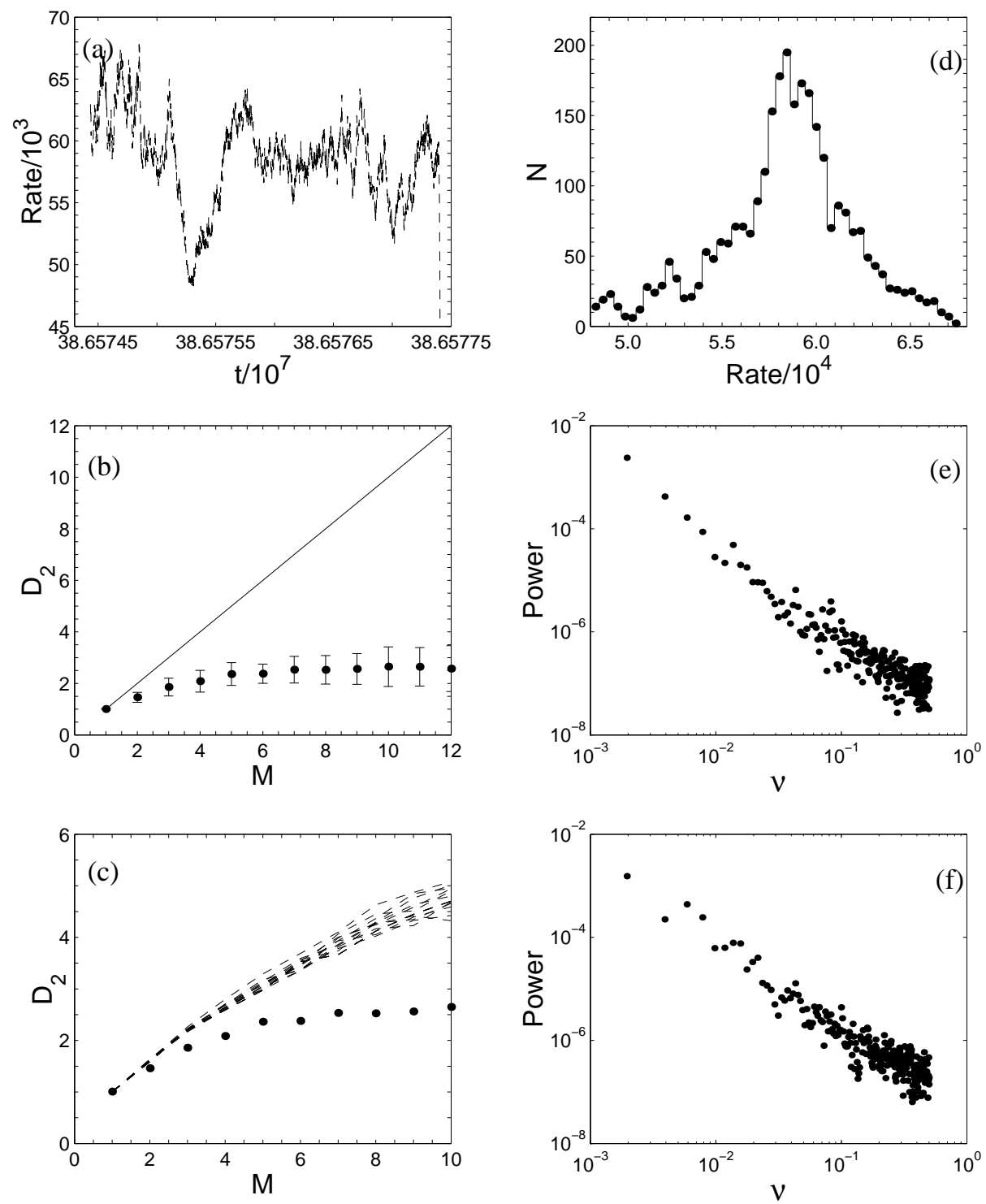

FIG. 2.- Sco X-1: (a) Variation of count rate as a function of time in units of $10^{7}$ sec (lightcurve), without subtracting the initial observation time. (b) Variation of correlation dimension, along with error bars, as a function of embedding dimension for original data. The solid line along the diagonal of the figure indicates an ideal stochastic curve. (c) Variation of correlation dimension as a function of embedding dimension for original (points) and corresponding surrogate (dashed lines) data. (d) Variation of number of count rate as a function of count rate itself in units of $10^{4} \mathrm{sec}^{-1}$ (Distribution) for original (solid line) and surrogate (points) data. Power-spectra for (e) original and (f) surrogate data. 
Search for chaos in neutron stars: Is Cyg X-3 a black hole?
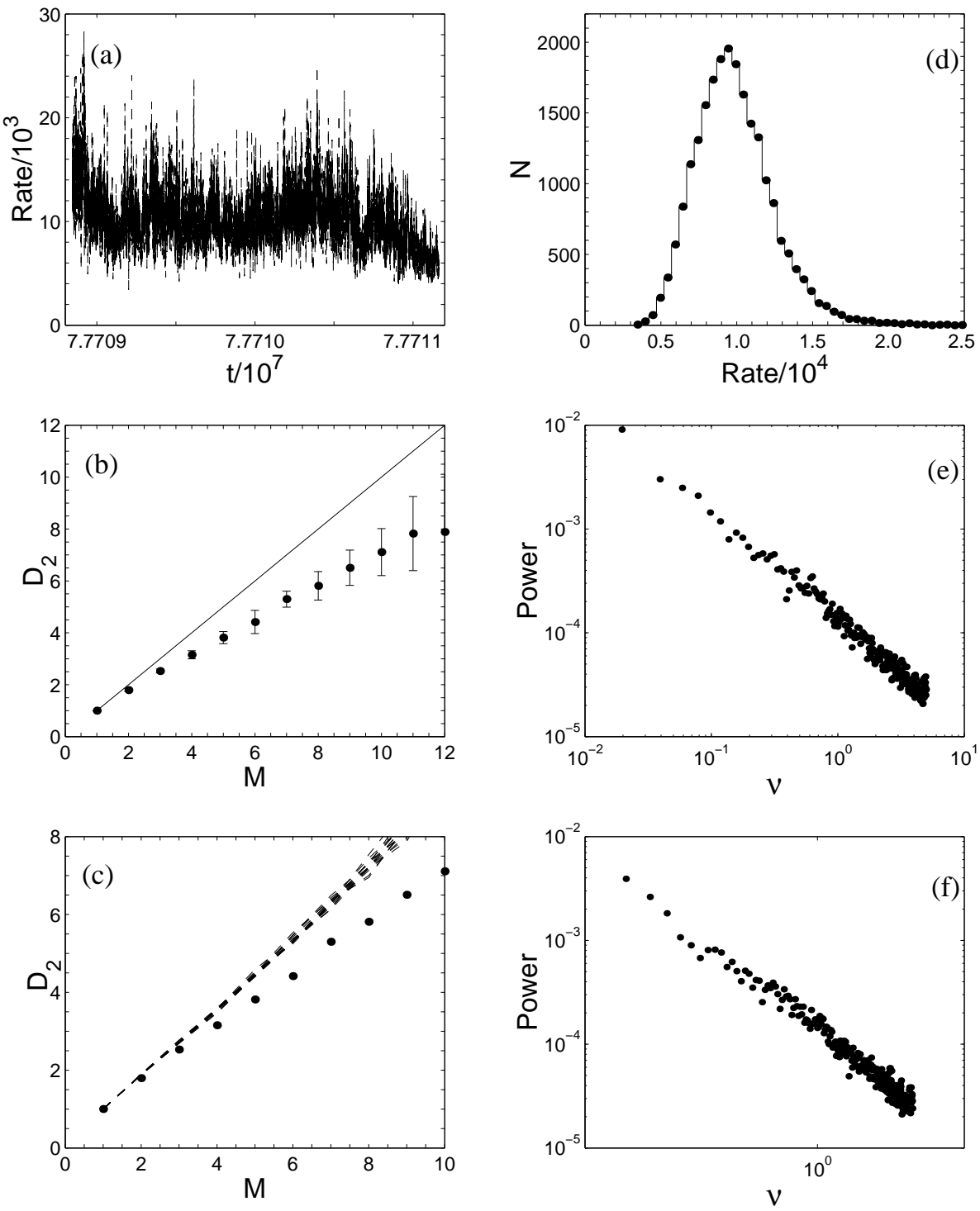

FIg. 3.- Cyg X-1: Same as Fig. 2 

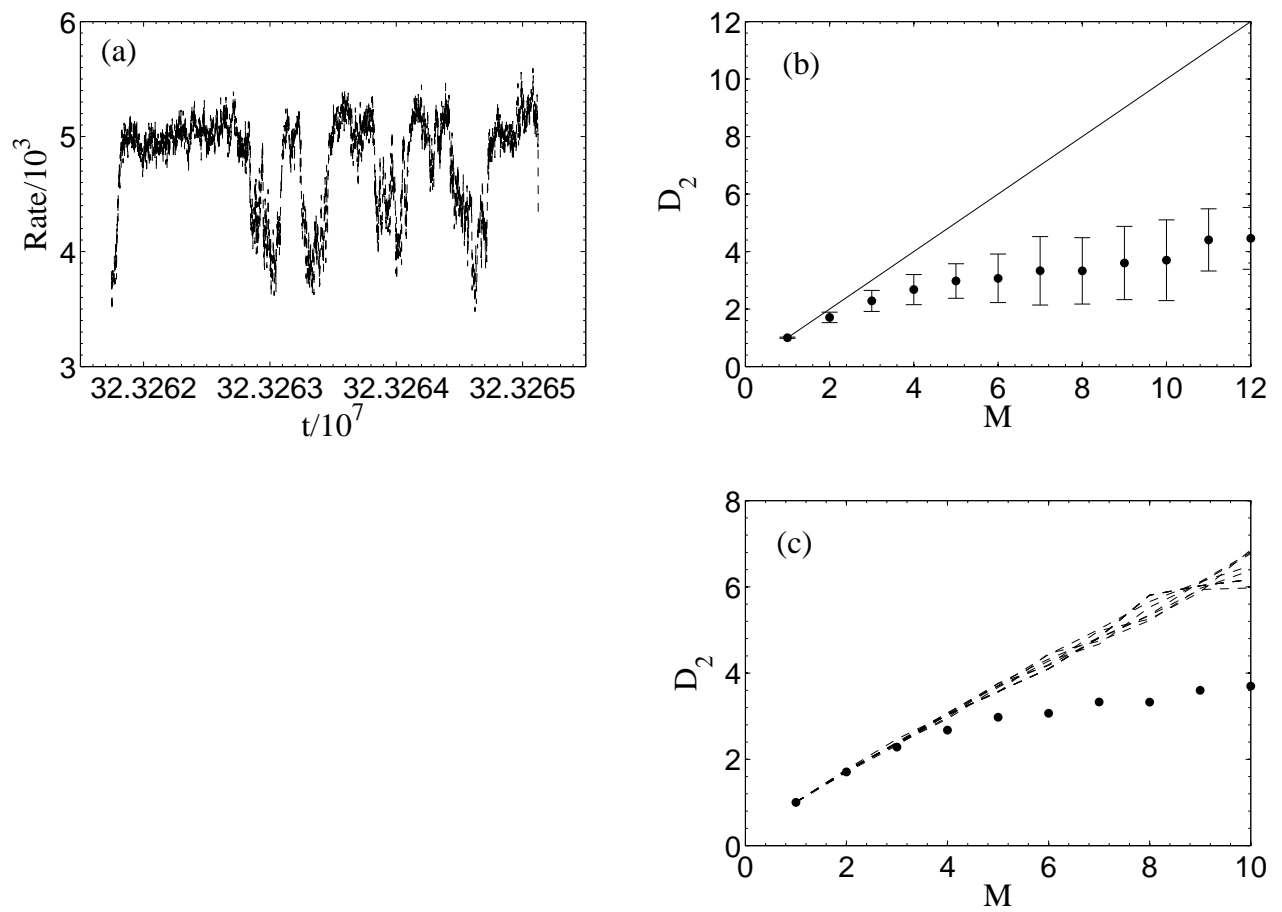

Fig. 4.- Cyg X-2: (a) Variation of count rate as a function of time in units of $10^{7} \mathrm{sec}$ (lightcurve). (b) Variation of correlation dimension, along with error bars, as a function of embedding dimension for original data. The solid line along the diagonal of the figure indicates an ideal stochastic curve. (c) Variation of correlation dimension as a function of embedding dimension for original (points) and corresponding surrogate (dashed lines) data.
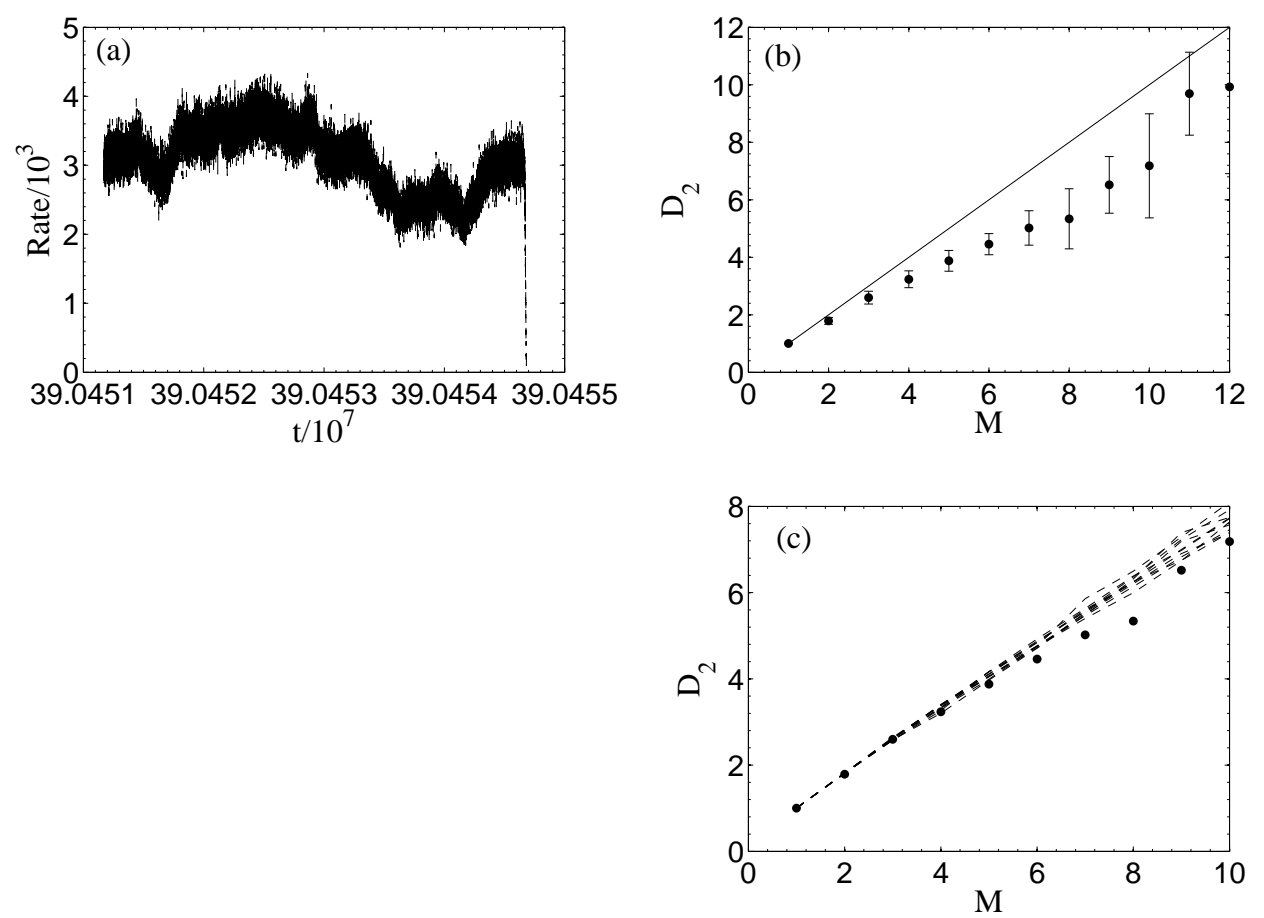

Fig. 5.- Cyg X-3: Same as Fig. 4 


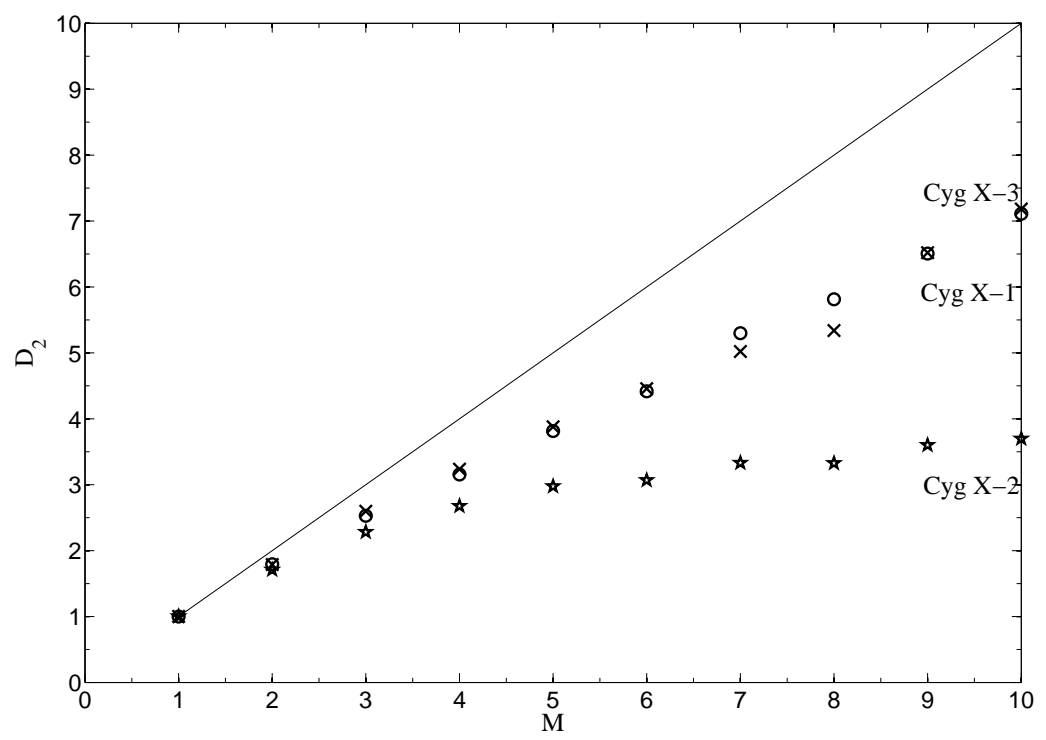

Fig. 6. - Comparison of the variation of correlation dimension as a function of embedding dimension between Cyg X-1 (open circle), Cyg X-2 (star), Cyg X-3 (cross). 